\title{
The Two Faces of Pharmacokinetics
}

\author{
Aldo Rescigno \\ Stillwater, MN, USA \\ Received, December 4, 2009; Revised, January 13, 2010; Accepted, January 22, 2010; Published, January 24, 2010.
}

\begin{abstract}
There are two main branches of Mathematics: Calculus and Geometry; in Physics there are Constructive Theories and Principle Theories. Similarly in Pharmacokinetics we can build models with two opposite approaches, the Bottom-Up and the Top-Down method. In this short opinion article I try to show, with the help of a few examples, the advantages of each one of the two approaches.
\end{abstract}

\section{CALCULUS AND GEOMETRY}

In my first year of graduate school I followed the lectures of a professor of Calculus and of a professor of Geometry; no two persons could have been more different. At that time, space travel was not yet fashionable, but if I had believed in it I would probably have said that the first Professor was from Jupiter and the second one from Mercury. On Jupiter a man weighs two or three times more than on Earth, so I guess he must move more slowly, has more time to think where to go, and when he reaches his destination he can stay there on very solid ground. On Mercury it is all the opposite; one feels lighter and can move fast, but must also decide quickly lest he falls into a trap.

I was completely captivated by my Calculus course; I could navigate through it with a minimum of effort and felt completely at ease with the logical connections of all its proofs. My feelings for the Geometry course were very different; even though I understood all theorems and their proofs, I could never or very seldom anticipate their logical connections. It was as though I had to follow a mysterious fairy; I trusted her, but could never predict her moves.

\section{CONSTRUCTIVE THEORIES PRINCIPLE THEORIES IN PHYSICS}

AND

A similar dichotomy is present in Physics, where we can distinguish two kinds of theories. With the first one we attempt to build a picture of more complex phenomena from a relatively simple formal scheme. Thus the kinetic theory of gases seeks to reduce mechanical, thermal, and diffusion processes to movements of molecules. When we say that we understand a group of natural phenomena, we invariably mean that a Constructive Theory has been found which covers the processes in question.

Along with the class of Constructive Theories there exists a second class, which can be called Principle Theories. These theories employ the analytic, not the synthetic, method. The elements forming their basis and starting-point are not hypothetically constructed but empirically discovered ones; from the general principles of the observed natural phenomena we can formulate the mathematical processes their theoretical representations have to satisfy. Thus the science of thermodynamics, starting from the universally experienced fact that perpetual motion is impossible, seeks, by analytical means, to deduce necessary conditions which separate events have to satisfy.

In the words of Einstein,[i] the advantages of the Constructive Theories are completeness, adaptability, and clearness; those of Principle Theories are logical perfection and security of the foundations.

\section{PHARMACOKINETICS}

How can we construct a model to help us understand what happens in a Pharmacokinetic experiment? The Calculus way or the Geometry way? Using a Constructive Theory or a Principle Theory?

Corresponding Author: Aldo Rescigno, 501 Main St. N. Ste 304, Stillwater, MN, U.S.A. E-Mail: rescigno@mac.com 
The dual aspects of Mathematics and of Physics are certainly present in Pharmacokinetics too. The two approaches that we can use in all pharmacokinetic problems could be called Bottomup and Top-down.

\section{Bottom-up}

The first approach to Pharmacokinetics started with a number of basic hypotheses; even though they were not always explicitly expressed, they were:

1. The substance administered to a subject is distributed among a number of compartments that can be recognized by their location and/or their chemical form;

2. All compartments are homogeneous;

3. The rate of elimination of substance by a compartment, or its rate of transfer from one compartment to another, is proportional to the amount of substance in the originating compartment.

In the case of a single compartment with bolus feeding those hypotheses lead to the mathematical model

$$
\text { (1) }\left\{\begin{array}{l}
\frac{d x}{d t}=-K x(t) \\
x(0)=D
\end{array}\right.
$$

where $K$ is the turnover rate of the compartment and $D$ is the bolus dose. The solution of the above differential equation is

$$
x(t)=D e^{-K t}
$$

an exponential function with negative slope.

This compartmental model can be expanded at will by connecting any number of compartments among them. With $n$ compartments we have $n$ first order differential equations that can be written in matrix notation

$$
\text { (2) } \frac{d \mathbf{X}}{d t}=-\mathbf{X}(t) \cdot \mathbf{K} \text {, }
$$

to be completed by appropriate initial conditions.
The row vector $\mathbf{X}(t)$ is formed by the functions $x_{1}(t), x_{2}(t), \ldots, x_{n}(t)$ of the individual compartments, while matrix $\mathbf{K}$ is formed by the turnover rates $K_{i}$ of compartments and transfer rates $k_{i j}$ between compartments, thus

$$
\mathbf{K}=\left(\begin{array}{cccc}
K_{1} & -k_{12} & \cdots & -k_{1 n} \\
-k_{21} & K_{2} & \cdots & -k_{2 n} \\
\vdots & \vdots & \ddots & \vdots \\
-k_{n 1} & -k_{n 2} & \cdots & K_{n}
\end{array}\right) .
$$

A through description of the properties of matrix $\mathbf{K}$ was published by Hearon.[ii] A number of pharmacokinetic parameters can be evinced from that matrix; for instance the inverse $\mathbf{T}=\mathbf{K}^{-1}$ of that matrix,

$$
\mathbf{T}=\left(\begin{array}{cccc}
t_{11} & t_{12} & \cdots & t_{1 n} \\
t_{21} & t_{22} & \cdots & t_{2 n} \\
\vdots & \vdots & \ddots & \vdots \\
t_{n 1} & t_{n 2} & \cdots & t_{n n}
\end{array}\right),
$$

if it exists, is formed by the permanence times $t_{i i}$ of compartment $i$ and by the residence times $t_{i j}$ from compartment $i$ to compartment $j$.[iii]

The hypotheses listed above can be modified in many ways. For instance hypothesis $\mathbf{3}$ can be substituted by the hypothesis that the substance in a particular compartment is eliminated by a process of order zero, or according to a catalyzed monomolecular irreversible reaction (MichaelisMenten kinetics). In all those cases the solution of the corresponding differential equations includes one or more terms that are not exponential; those cases may still be tractable, though not as simply as with the standard hypothesis 3.[iv]

Modifying hypothesis 2 implies more serious complications. In a non-homogeneous compartment the rates of elimination and of transfer do not depend upon the total amount of substance in the compartment, but upon its concentration at various locations inside the compartment. The concentration 
itself is a function of time and of location; the ordinary differential equations (1) and (2) must be substituted by partial differential equations. They must be solved case by case. Of course, all hypotheses need to be confirmed by appropriate experimental evidence.[v]

According to Einstein's statement quoted before, the bottom-up approach to Pharmacokinetics is a principle theory, and as such it has the advantages of logical perfection and security of foundations; it has been used for many years to investigate and describe the behavior of many drugs.

Why many pharmacokineticists at one point felt the need to elaborate a different approach to Pharmacokinetics? One reason, the one most frequently cited, was that the constants $K_{i}$ and $k_{i j}$ contained in matrix $\mathbf{K}$ of equation (2) were not always given a physiological interpretation. The indiscriminate use of software packages allowing the fitting of experimental data with sums of a very large number of exponential functions, contributed to the need for a different approach. The goodness of fitting was prevailing upon the physiological meaning.

\section{Top-down}

The bottom-up approach to pharmacokinetic model building started from first principles, using differential equations; the top-down approach started from end results, using integral equations.

The top-down approach, often called physiological modeling, was a constructive theory, and as such had the advantages of completeness, adaptability, and clearness. Clearness, in particular, made it the method of choice in many cases when the available data were not sufficiently detailed to allow the computation of many pharmacokinetic parameters.

If $f(t)$ measures the concentration of a substance in a certain point of a subject, and $h(t)$ the concentration of the same substance or one of its products in a point where it has been transferred, we can write the integral equation

$$
\int_{0}^{t} f(\tau) g(t, \tau) d \tau=h(t)
$$

This is a Volterra integral equation of the second type; function $g(t)$, called kernel of the equation, describes the transport of the substance of interest from one point, the precursor, to another point, the product. The expression $g(t, \tau) d \tau$ can be interpreted as the probability that a particle that left the precursor in the interval of time from $\tau$ to $\tau+$ $d \tau$ will be in the product at time $t$.[vi]

The solution of this equation implies difficulties of a high order, unless we make the additional hypothesis that its kernel is a function of one variable only, i. e., that

$$
g(t, \tau)=g(t-\tau)
$$

This hypothesis means that the probability of transfer from precursor to product depends upon the interval of time between the two observations and not on the absolute time; in other words, this transfer is time-invariant. Function $g(t-\tau)$ is called transfer function from precursor $f(t)$ to product $h(t)$.

The simpler form of the Volterra integral equation,

$$
\int_{0}^{t} f(\tau) g(t-\tau) d \tau=h(t)
$$

is called convolution of function $f(t)$ and $g(t)$.

The use of transfer functions and convolutions in Pharmacokinetics started many years ago.[vii]

From the convolution equation, by integration of both sides from 0 to $\infty$, we get

(3) $\int_{0}^{\infty} f(t) d t \cdot \int_{0}^{\infty} g(t) d t=\int_{0}^{\infty} h(t) d t$.

If we multiply both sides of the convolution equation by $d t$, then integrate as before, we get

$$
\int_{0}^{\infty} t f(t) d t \cdot \int_{0}^{\infty} g(t) d t+\int_{0}^{\infty} f(t) d t \cdot \int_{0}^{\infty} t g(t) d t=\int_{0}^{\infty} t h(t) d t
$$

this equation can be written in the form

$$
\text { (4) } \frac{\int_{0}^{\infty} t f(t) d t}{\int_{0}^{\infty} f(t) d t}+\frac{\int_{0}^{\infty} t g(t) d t}{\int_{0}^{\infty} g(t) d t}=\frac{\int_{0}^{\infty} t h(t) d t}{\int_{0}^{\infty} h(t) d t} \text {. }
$$

Both equations (3) and (4) have an important phenomenological interpretation; see for instance Rescigno [viii] and Rescigno and Gurpide.[ix] 
The two expressions $\int_{0}^{\infty} c(t) d t$ and $\int_{0}^{\infty} t c(t) d t / \int_{0}^{\infty} c(t) d t$, where $c(t)$ is the concentration of a given substance at a generic point of a subject, have been frequently used in Pharmacokinetics, with various interpretations. I shall examine here just a few possible interpretations.

\section{Availability}

According to the Food and Drug Administration, $[\mathrm{x}]$ "Bioavailability means the rate and extent to which the active ingredient or active moiety is absorbed from a drug product and becomes available at the site of action." This description of Bioavailability cannot easily be quantified; while the extent of absorption may be defined as the ratio between amount of drug absorbed and amount delivered, the rate may not be unique if the absorption is not a simple onecompartment distribution without recycling.

On second thought, what really matters is not the rate of absorption per se, but how much drug is present at the site of action and when it is there; in short the function $c(t)$ tells us all we need to know, i. e., how much is available at the site of action and when. Thus the Bioavailability can be easily quantified not as a single parameter, but as a function of time, by defining it as the fraction $c(t) / D$.

If we make the two additional hypotheses that the effect of the drug is proportional to its concentration at the site of action and to the duration of its presence there, and that the effects at different intervals of time add linearly, we can say that the integral $\int_{0}^{\infty} c(t) d t / D$ is a measure of the exposure of a drug.

\section{Clearance}

If a drug is eliminated from an organ with a uniform concentration $c(t)$, by definition of clearance $C l$ we can write $C l \cdot c(t)=r(t)$, where $r(t)$ is the rate of elimination from that organ. If we multiply by $d t$ and integrate from 0 to $\infty$ we get

$$
\int_{0}^{\infty} C l \cdot c(t) d t=\int_{0}^{\infty} r(t) d t
$$

but the integral at the right-hand side is the total drug eliminated; we can call it $Q$. If the clearance is constant over time, we can export it from the lefthand side integral to get

$$
\int_{0}^{\infty} c(t) d t=\frac{Q}{C l} .
$$

This formula can be used to compute the clearance when the clearance is constant over time and the concentration in the organ is uniform. In all other cases that formula is approximate, and the approximation depends upon the deviation of the experimental conditions from the necessary hypotheses stated above.[xi]

\section{Exit Time and Transfer Time}

The ratio $\int_{0}^{\infty} t c(t) d t / \int_{0}^{\infty} c(t) d t$ can be given different interpretations. Without any additional hypotheses this ratio is the average age of the drug at the point of sampling; in other words it is the average time elapsed from the beginning of the experiment to the presence of the drug at the point of sampling.

If we make the hypothesis that the clearance in a definite volume around the sampling point is constant in time, we can write the identity

$$
\frac{\int_{0}^{\infty} t \cdot c(t) d t}{\int_{0}^{\infty} C(t) d t}=\frac{\int_{0}^{\infty} t \cdot C l \cdot c(t) d t}{\int_{0}^{\infty} C l \cdot c(t) d t}
$$

but the product $\mathrm{Cl} \cdot \mathrm{c}(t) d t$ is the amount of drug cleared in the interval of time from $t$ to $t+d t$, and $t$ is the time of this event; therefore the above ratio is the average time when the drug is cleared from the sampling point. This time is called exit time, symbol $\Omega:[\mathrm{xii}]$

$$
\Omega=\frac{\int_{0}^{\infty} t \cdot c(t) d t}{\int_{0}^{\infty} c(t) d t} .
$$

Looking at equation (4), if the hypotheses made above are valid for both precursor and product, when we sample the concentration $f(t)$ of a precursor and $h(t)$ of its product, the difference 


$$
\frac{\int_{0}^{\infty} t \cdot g(t) d t}{\int_{0}^{\infty} g(t) d t}=\frac{\int_{0}^{\infty} t \cdot h(t) d t}{\int_{0}^{\infty} h(t) d t}-\frac{\int_{0}^{\infty} t \cdot f(t) d t}{\int_{0}^{\infty} f(t) d t}
$$

may be called transfer time; it measures the average time taken by the drug to transfer from precursor products.

\section{CONCLUSION}

The top-down approach has certainly the advantages of adaptability and clearness; what makes it particularly attractive, is its ability to connect some simple geometrical properties, like area under a curve, to well known physiological properties, like clearance and availability; and this may be done with a minimum of mathematical speculation.

It hides a possible danger, though, what the logicians call petitio principii, of assuming as a true premise a proposition that is yet to be proved. The following remarks should clarify this point.

Model building always goes through two phases; they are what the French call histoire and récit.[xiii] The first phase or histoire, is the uncritical description of the observed facts. Of course, we observe only the facts that we consider relevant to a problem, therefore there is never a completely uncritical collection of observations; nevertheless in the histoire the mere description of the experimental facts is prevalent, and it is presented as a temporal succession of facts, without any cause-effect relationship.

The second phase or récit, is not any more an objective description of facts, but a subjective interpretation of the histoire; the cause-effect relationship is prevalent and all facts are logically connected among them.

If you observe carefully the few examples I presented, it should be evident that the hypotheses necessary for the transition from histoire to récit in the top-down approach to Pharmacokinetics are exactly the same hypotheses that were used in the bottom-up approach. The inescapable conclusion of all modeling is: "Use the bottom-up or the topdown approach, whichever you are more comfortable with; but in either case the same fundamental hypotheses are necessary for your conclusions to have any physiological meaning."

\section{REFERENCES}

1. Einstein A. What is the theory of relativity? The London Times. November 28, 1919.

2. Hearon JZ. Theorems on Linear Systems. Ann N Y Acad Sci 1963; 108: 36-58.

3. Rescigno A. Compartmental Analysis and Its Manifold Applications to Pharmacokinetics. AAPS Journal 2010; 12: 61-72 DOI: 10.1208/s12248-0099160-x.

4. Rescigno A. Foundations of Pharmacokinetics. New York: Kluwer Academic/Plenum Publishers; 2003. Pages 127-134.

5. Rescigno A, Beck JS. The Use and Abuse of Models. J Pharmacokin Biopharm 1987; 15: 327340.

6. Rescigno A, Segre G. Analysis of Multicompartmented Biological Systems. J theoret Biol 1962; 3: 149-163.

7. Rescigno A, Segre G. La Cinetica dei Farmaci e dei Traccianti Radioattivi. Torino: Paolo Boringhieri; 1961. English translation: Drug and tracer Kinetics. Waltham: Blaisdell; 1966.

8. Rescigno A. On Transfer Times in Tracer Experiments. J theoret Biol 1973; 39: 9-27.

9. Rescigno A, Gurpide E. Estimation of Average Times of Residence, Recycle, and Interconversion of Blood-Borne Compounds Using Tracer Methods. J Clin Endocrinol Metab 1973; 36: 263-276.

10. Code of Federal Regulations, Title 21, Volume 5, Revised as of April 1, 2009. U.S. Government Printing Office via GPO Access [CITE: 21CFR320.1].

11. Rescigno A, Marzo A. Area Under the Curve, Bioavailability, and Clearance. J Pharmacokin Biopharm 1991; 19: 473-482.

12. Mordenti J, RescignoA. Estimation of Permanence Time, Exit Time, Dilution Factor, and Steady-State Volume of Distribution. Pharm Res 1992; 9: 17-25.

13. Benveniste $E$. Problèmes de linguistique générale, tome 1. Paris: Tel Gallimard; 1966. 
